\title{
COAST-ES: Herramienta integral de tamizaje para adultos mayores ${ }^{1}$
}

\author{
Karima Alabasi, Nancy J. Gal y Wendy J. Dahl; traducido por Daniela Rivero Mendoza²
}

La malnutrición resulta de la falta de adecuada nutrición para mantener la buena salud (Moreira et al. 2016). De particular preocupación en los adultos mayores es la malnutrición resultante de proteínas y energía (calorías) inadecuadas que conducen a la pérdida muscular (Crichton et al. 2019). El tamizaje (cribado) del riesgo nutricional es un primer paso para identificar rápidamente a las personas que pueden estar malnutridas o en riesgo de malnutrición. Una herramienta de tamizaje de detección de malnutrición debe ser fácil de usar, rápida de administrar y válida para poder identificar correctamente a las personas en riesgo de malnutrición. La Herramienta Integral de Tamizaje de Adultos Mayores (COAST, por sus siglas en inglés) es una herramienta válida y práctica para determinar si los adultos mayores que viven en la comunidad están en riesgo de malnutrición (Alabasi et al. 2018). Para el cribado de malnutrición con COAST, se considera que los adultos mayores son aquellas personas mayores de 60 años.

Existe una alta prevalencia de malnutrición entre los adultos mayores que viven en la comunidad, particularmente en las comunidades rurales (Crichton et al. 2019). La malnutrición es un problema de salud importante, ya que está asociada con la fragilidad (Lorenzo-Lopez et al. 2017), la reducción de la calidad de vida (Rasheed y Woods 2013) y la mortalidad (Shakersain et al. 2016). Los adultos mayores que reciben servicios de comidas congregadas y entregadas en el hogar tienen un mayor riesgo de malnutrición que la población general adulta mayor de los EE. UU., debido a su precario estado de salud, funcional y financiero (Lloyd y Wellman 2015).

La pérdida de peso involuntaria resulta de no consumir suficientes calorías y puede conducir a la malnutrición. Además, muchos adultos mayores pueden no consumir proteínas adecuadas, lo que los pone en riesgo de malnutrición (ter Borg et al. 2015). Evidencia de alto nivel apoya que la falta de apetito, así como la hospitalización, la dependencia alimentaria, la mala salud autopercibida y el mal funcionamiento físico son determinantes de malnutrición (O’Keeffe et al. 2018). Un estudio reciente confirmó que el riesgo de malnutrición aumenta en adultos mayores con poco apetito y dificultades para comer, así como también en aquellos con enfermedades respiratorias y gastrointestinales (Kiesswetter et al. 2020). La herramienta COAST fue diseñada para enfocarse en estos factores de riesgo con preguntas sobre pérdida de peso involuntaria, apetito, cambios en el tipo y/o cantidad de alimentos consumidos debido a enfermedades y condiciones de salud, y la ingesta de alimentos con proteínas. Aunque la calidad de la dieta no está asociada con la malnutrición proteico-energética (Hengeveld et al. 2018), la pregunta “¿Qué tan saludable es su dieta general?” se agregó como representante de la calidad de la dieta, específicamente el consumo de frutas

1. Este documento, FSHN20-37s, es uno de una serie de publicaciones del Food Science and Human Nutrition, Servicio de Extensión Cooperativa de la Florida, Instituto de Alimentos y Ciencias Agrícolas, Universidad de la Florida. (UF/IFAS). Fecha de primera publicación: julio 2020. Visite nuestro sitio web EDIS en https://edis.ifas.ufl.edu.

2. Karima Alabasi, former doctoral student, Food Science and Human Nutrition Department, UF/IFAS Extension; Nancy J. Gal, Extension agent IV, UF/IFAS Extension Marion County; and Wendy J. Dahl, associate professor, Food Science and Human Nutrition Department, UF/IFAS Extension, Gainesville, FL 32611. Traducido por Daniela Rivero Mendoza.

The Institute of Food and Agricultural Sciences (IFAS) is an Equal Opportunity Institution authorized to provide research, educational information and other services

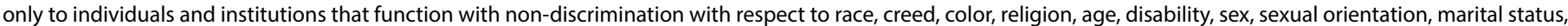

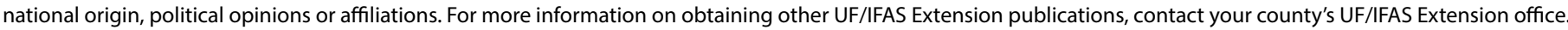
U.S. Department of Agriculture, UF/IFAS Extension Service, University of Florida, IFAS, Florida A \& M University Cooperative Extension Program, and Boards of County Commissioners Cooperating. Nick T. Place, dean for UF/IFAS Extension. 
y verduras, ya que esto puede ser un fuerte indicador del consumo de vitaminas y minerales (Loftfield et al. 2015).

Existen muchas herramientas de evaluación de malnutrición y algunas están destinadas a adultos mayores que viven en la comunidad (Isautier et al. 2019). Una de esas herramientas, la Herramienta de Tamizaje de Malnutrición (MST, por sus siglas en inglés), se ha recomendado recientemente para el tamizaje de malnutrición en todos los adultos, independientemente de su edad y en todos los entornos (Skipper et al. 2020). Sin embargo, su baja especificidad en adultos mayores en entornos comunitarios es preocupante (Dwyer et al. 2019). Además, se basa en una estimación de pérdida de peso que puede no conocerse y ofrece desafíos logísticos para la medición en un entorno comunitario. Además, el formulario corto del Mini Nutrition Assessment ( $\mathrm{MNA}^{\oplus}$-SF) se usa comúnmente para detectar la desnutrición en entornos clínicos, pero requiere medir la altura y el peso, o alternativamente la circunferencia de la pantorrilla, que puede llevar mucho tiempo y ser difícil de realizar en la comunidad.

La herramienta COAST fue diseñada para ser breve. Tiene solo cinco preguntas y puede administrarse mediante entrevista o auto tamizaje. La herramienta COAST se desarrolló específicamente para adultos mayores de bajo nivel socioeconómico con alto riesgo de malnutrición. Esto contrasta con otras herramientas de detección dirigidas a la población general de adultos mayores (Morrison, Laur y Keller 2019).

Es necesario identificar a los adultos mayores que corren el riesgo de sufrir malnutrición para garantizar que reciban las recomendaciones adecuadas para los servicios de alimentación y nutrición. Dado que la herramienta COAST se desarrolló para el tamizaje en sitios de comidas congregadas y otros grupos de adultos mayores de escasos recursos, la herramienta puede ser ideal para la evaluación de resultados a largo plazo de los programas de educación nutricional de Extensión dirigidos a estas poblaciones. 
Primer nombre: Apellido: Fecha:

1.¿Ha perdido peso usted recientemente sin intentarlo? (Ferguson et al. 1999)

$$
\begin{aligned}
& 0 \text { = Sí } \\
& 1 \text { = No }
\end{aligned}
$$

2. ¿Ha estado usted comiendo menos alimentos debido a una disminución de apetito? (Ferguson et al. 1999)

$$
\begin{aligned}
& 0=\text { Sí } \\
& 1=\text { No }
\end{aligned}
$$

3. ¿Tiene usted una enfermedad o condición que le ha hecho cambiar el tipo y la cantidad de alimentos que come? (Nutrition Screening Initiative [NSI] 1994)

$$
\begin{aligned}
& 0=\text { Sí } \\
& 1=\text { No }
\end{aligned}
$$

4. En general, ¿Qué tan saludable es su dieta? (Loftfield et al. 2015)

$0=$ Pobre

$1=$ Buena

$2=$ Muy buena

5. ¿Usted consume...

- Productos lácteos (leche, queso, yogur) o leche de soya al menos una vez al día?

- Carne, ave de corral (p.ej. pollo), pescado/mariscos, o huevos todos los días?

- Legumbres (p.ej. frijoles), productos de soya, nueces o semillas al menos dos veces por semana? (Vellas et al. 1999)

$0=$ Si no respondió ningún sí

1 = Si respondió un sí

$2=$ Si respondió dos sí

$3=$ Si respondió tres sí

\section{Puntuación (subtotal máximo 8 puntos)}

7-8 puntos: $\square$ Bajo riesgo de malnutrición

5-6 puntos: $\square$ Riesgo moderado de malnutrición

0-4 puntos: $\square$ Alto riesgo de malnutrición 


\section{COAST - ES}

1. ¿Ha perdido peso usted recientemente sin intentarlo? (Ferguson et al. 1999)

Sí

No

2. ¿Ha estado usted comiendo menos alimentos debido a una disminución de apetito? (Ferguson et al. 1999)

Sí

No

3. ¿Tiene usted una enfermedad o condición que le ha hecho cambiar el tipo y la cantidad de alimentos que come? (Nutrition Screening Initiative [NSI] 1994)

Sí

No

4. En general, ¿Qué tan saludable es su dieta? (Loftfield et al. 2015)

Pobre

Buena

Muy buena

5. ¿Usted consume...

- Productos lácteos (leche, queso, yogur) o leche de soya al menos una vez al día?

Sí

No

- Carne, ave de corral (p.ej. pollo), pescado/mariscos, o huevos todos los días?

Sí

No

- Legumbres (p.ej. frijoles), productos de soya, nueces o semillas al menos dos veces por semana?(Vellas et al. 1999) Sí

No 


\section{References}

Alabasi, K., N. Gal, A. Fatani, L. Mead, and W. Dahl. 2018. "Development and Validation of a Comprehensive Older Adult Screening Tool (COAST): A Practical Tool for Identifying Malnutrition Risk." Journal of the Academy of Nutrition and Dietetics 118 (9): A27. https://doi. org/10.1016/j.jand.2018.06.281.

Crichton, M., D. Craven, H. Mackay, W. Marx, M. de van der Schueren, and S. Marshall. 2019. "A Systematic Review, Meta-analysis and Meta-regression of the Prevalence of Protein-Energy Malnutrition: Associations with Geographical Region and Sex." Age and Ageing 48 (1): 38-48. https:// doi.org/10.1093/ageing/afy144.

Dwyer, J. T., J. J. Gahche, M. Weiler, and M. B. Arensberg. 2019. "Screening Community-Living Older Adults for Protein Energy Malnutrition and Frailty: Update and Next Steps." Journal of Community Health 45: 640-660. https:// doi.org/10.1007/s10900-019-00739-1.

Ferguson, M., S. Capra, J. Bauer, and M. Banks. 1999. “Development of a Valid and Reliable Malnutrition Screening Tool for Adult Acute Hospital Patients." Nutrition 15 (6): 458-64. https://doi.org/10.1016/s0899-9007(99)00084-2.

Hengeveld, L. M., H. A. H. Wijnhoven, M. R. Olthof, I. A. Brouwer, T. B. Harris, S. B. Kritchevsky, A. B. Newman, and M. Visser. 2018. "Prospective Associations of Poor Diet Quality with Long-Term Incidence of Protein-Energy Malnutrition in Community-Dwelling Older Adults: The Health, Aging, and Body Composition (Health ABC) Study." American Journal of Clinical Nutrition 107 (2): 155-164. https://doi.org/10.1093/ajcn/nqx020.

Isautier, J. M. J., M. Bosnic, S. S. Y. Yeung, M. C. Trappenburg, C. G. M. Meskers, A. C. Whittaker, and A. B. Maier. 2019. "Validity of Nutritional Screening Tools for Community-Dwelling Older Adults: A Systematic Review and Meta-analysis." Journal of the American Medical Directors Association 20 (10): 1351.e13-1351.e25. https:// doi.org/10.1016/j.jamda.2019.06.024.

Kiesswetter, E., M. G. Colombo, C. Meisinger, A. Peters, B. Thorand, R. Holle, K. H. Ladwig, H. Schulz, E. Grill, R. Diekmann, E. Schrader, P. Stehle, C. C. Sieber, and D. Volkert. 2020. "Malnutrition and Related Risk Factors in Older Adults from Different Health-Care Settings: An Enable Study." Public Health Nutr. 23 (3): 446-456. https:// doi.org/10.1017/s1368980019002271.
Lloyd, J. L., and N. S. Wellman. 2015. "Older Americans Act Nutrition Programs: A Community-Based Nutrition Program Helping Older Adults Remain at Home." Journal of Nutrition in Gerontology and Geriatrics 34 (2): 90-109. https://doi.org/10.1080/21551197.2015.1031592.

Loftfield, E., S. Yi, S. Immerwahr, and D. Eisenhower. 2015. "Construct Validity of a Single-Item, Self-Rated Question of Diet Quality." Journal of Nutrition Education and Behavior 47 (2): 181-7. https://doi.org/10.1016/j.jneb.2014.09.003.

Lorenzo-Lopez, L., A. Maseda, C. de Labra, L. RegueiroFolgueira, J. L. Rodriguez-Villamil, and J. C. Millan-Calenti. 2017. "Nutritional Determinants of Frailty in Older Adults: A Systematic Review." BMC Geriatrics 17 (1): 108. https:// doi.org/10.1186/s12877-017-0496-2.

Moreira, N. C., S. Krausch-Hofmann, C. Matthys, C. Vereecken, E. Vanhauwaert, A. Declercq, G. E. Bekkering, and J. Duyck. 2016. "Risk Factors for Malnutrition in Older Adults: A Systematic Review of the Literature Based on Longitudinal Data." Adv. Nutr. 7 (3): 507-22. https://doi. org/10.3945/an.115.011254.

Morrison, J. M., C. V. Laur, and H. H. Keller. 2019. "SCREEN III: Working towards a Condensed Screening Tool to Detect Nutrition Risk in Community-Dwelling Older Adults Using CLSA Data." European Journal of Clinical Nutrition 73 (9): 1260-1269. https://doi.org/10.1038/ s41430-019-0411-3.

Nutrition Screening Initiative. 1994. “The Determine Your Nutritional Health Checklist.” Wash. Nurse 24 (2): 14-15.

O’Keeffe, M., M. Kelly, E. O’Herlihy, P. W. O’Toole, P. M. Kearney, S. Timmons, E. O'Shea, C. Stanton, M. Hickson, Y. Rolland, C. Sulmont Rosse, S. Issanchou, I. Maitre, M. Stelmach-Mardas, G. Nagel, M. Flechtner-Mors, M. Wolters, A. Hebestreit, L. C. P. G. M. De Groot, O. van de Rest, R. Teh, M. A. Peyron, D. Dardevet, I. Papet, K. Schindler, M. Streicher, G. Torbahn, E. Kiesswetter, M. Visser, D. Volkert, and E. M. O’Connor. 2018. "Potentially Modifiable Determinants of Malnutrition in Older Adults: A Systematic Review." Clinical Nutrition 38 (6): 2477-2498. https:// doi.org/10.1016/j.clnu.2018.12.007.

Rasheed, S., and R. T. Woods. 2013. "Malnutrition and Quality of Life in Older People: A Systematic Review and Meta-analysis." Ageing Research Reviews 12 (2): 561-6. https://doi.org/10.1016/j.arr.2012.11.003. 
Shakersain, B., G. Santoni, G. Faxen-Irving, D. Rizzuto, L. Fratiglioni, and W. Xu. 2016. "Nutritional Status and Survival among Old Adults: An 11-Year Population-Based Longitudinal Study." European Journal of Clinical Nutrition 70 (3): 320.

Skipper, A., A. Coltman, J. Tomesko, P. Charney, J. Porcari, T. A. Piemonte, D. Handu, and F. W. Cheng. 2020. "Position of the Academy of Nutrition and Dietetics: Malnutrition (Undernutrition) Screening Tools for All Adults." Journal of the Academy of Nutrition and Dietetics 120 (4): 709-713. https://doi.org/10.1016/j.jand.2019.09.011.

ter Borg, S., S. Verlaan, D. M. Mijnarends, J. M. G. A. Schols, L. C. P. G. M. De Groot, and Y. C. Luiking. 2015. "Macronutrient Intake and Inadequacies of CommunityDwelling Older Adults, a Systematic Review." Annals of Nutrition and Metabolism 66 (4): 242-255. https://doi. org/10.1159/000435862.

Vellas, B., Y. Guigoz, P. J. Garry, F. Nourhashemi, D. Bennahum, S. Lauque, and J. L. Albarede. 1999. “The Mini Nutritional Assessment (MNA) and Its Use in Grading the Nutritional State of Elderly Patients." Nutrition 15 (2): 116-22. https://doi.org/10.1016/s0899-9007(98)00171-3. 